\title{
AN SLS4 LONGITUDE SYSTEM BASED ON A TRACKING FILTER ANALYSIS OF THE ROTATIONAL MODULATION OF SATURN KILOMETRIC RADIATION
}

\author{
D. A. Gurnett*, J. B. Groene*, T. F. Averkamp*, W. S. Kurth*, \\ S.-Y. Ye*, and G. Fischer ${ }^{\dagger}$
}

\begin{abstract}
Saturn has been known for over thirty years to emit an intense radio emission at kilometer wavelengths called Saturn Kilometric Radiation (SKR) that is modulated by the rotation of the planet. Although the period of this modulation was initially thought to represent the rotation period of the planet, it is now known that the radiation has two distinctly different rotational modulation periods that vary by on the order of one percent on times scales of years. One component originates primarily from the northern auroral region, and the other originates primarily from the southern auroral region. The differences in the modulation periods are believed to be due to latitudinal variations in the slippage of the magnetosphere relative to the interior of planet, apparently controlled by the seasonal variation in the tilt of Saturn's rotational axis. Since other magnetospheric phenomena display similar complicated rotational modulation effects, there is a need to define north and south longitude systems based on the variable SKR modulation periods in the two hemispheres. Because the SKR signal received by the spacecraft often includes both components it is sometimes difficult to separate the phases of the two components. In this paper we describe a method of determining the two phases based on a tracking filter approach that can separately track the modulation waveforms of the two components. The phases of the two waveforms can then be used to define a new longitude system for the northern and southern components that we call the SLS4 longitude system. This is an extension of the previous SLS2 and SLS3 longitude systems, which only described phase variations of the southern component.
\end{abstract}

* Department of Physics and Astronomy, University of Iowa, Iowa City, IA 52242, USA

† Space Research Institute, Austrian Academy of Sciences, Schmiedlstrasse 6, A-8042 Graz, Austria 


\section{Introduction}

Saturn kilometric radiation, often abbreviated SKR, is an intense radio emission at kilometer wavelengths that originates from the high-latitude auroral regions of Saturn's magnetosphere. First discovered during the 1980-1981 Voyager 1 and 2 flybys of Saturn [Kaiser et al., 1980], it was soon shown that the radio emission exhibited a clock-like modulation at a period of $10 \mathrm{~h} 39 \mathrm{~m} 24 \pm 7 \mathrm{~s}$ that was interpreted as the rotation period of Saturn [Desch and Kaiser, 1981]. Because the rotation period of Saturn cannot be determined by optical means due to the motion of clouds, this period was formally adopted as the rotation period of Saturn [Davies et al., 1996] and now provides the basis for the Saturn Longitude System (SLS) defined by the International Astronomical Union (IAU) [Seidelmann et al., 2002].

Although the rotation period obtained from the relatively brief Voyager flybys was widely accepted as the rotation period of Saturn, radio measurements by the Ulysses spacecraft in the late 1990s showed that the SKR modulation period varied by up to one percent on time scales of years [Lecacheux et al., 1997; Galopeau and Lecacheux, 2000]. These long term variations were subsequently confirmed by radio observations from the Cassini spacecraft during the approach to Saturn in 2002 to 2004 which showed that the modulation period had increased by approximately 6 minutes from the Voyager era [Gurnett et al., 2005]. After Cassini was placed into orbit around Saturn on 1 July 2004 it was soon discovered that the SKR has two distinct modulation periods [Kurth et al., 2008], and that the first, with a period of about $10.8 \mathrm{~h}$, originates primarily from a source in the southern auroral region, and that the second, newly discovered component, with a period of about $10.6 \mathrm{~h}$, originates from a source in the northern auroral region [Gurnett et al., 2009]. These two periods have since converged and reversed about seven months after the 11 August 2009 equinox, and a similar reversal was identified in the Ulysses data about nine months after the previous 19 November 1995 equinox [Gurnett et al., 2010]. Thus, the long term variations in the modulation rate of the two components are apparently controlled by the changing tilt of Saturn's rotational axis relative to the sun, most likely due to seasonal north-south differences in the ionospheric conductivity and high-altitude zonal winds that drive the rotation of the magnetosphere.

It soon became apparent after Cassini arrived at Saturn that a longitude system was needed to organize magnetospheric plasma and magnetic field data. To satisfy this need Kurth et al. [2007] introduced a new longitude system, called SLS2, valid from 1 January 2004 to 28 August 2006, that was based on the rotational modulation rate of the first (south) component. The SLS2 longitude system was subsequently refined and extended to 10 August 2007 by Kurth et al. [2008] and is called the SLS3 longitude system. The method used by Kurth et al. [2007, 2008] to track the variable SKR modulation rate was to assume a constant rotational modulation rate, $\omega$, and then make a plot of the intensity as a function of the subsolar longitude, $\lambda_{\text {Sun }}=\omega\left(\mathrm{t}-\mathrm{t}_{0}\right)$ and time, $\mathrm{t}$. In this plot any variation from a constant rotation rate appears as a drift in the longitude of the peak SKR intensity as a function of time, see Figure 1. The drift pattern indicated by the upward curving white line is due to the modulation of the first (south) SKR component. The drift pattern indicated by the much steeper downward sloping white line is due the modulation of the second (north) component, discovered by Kurth et al. [2008]. 


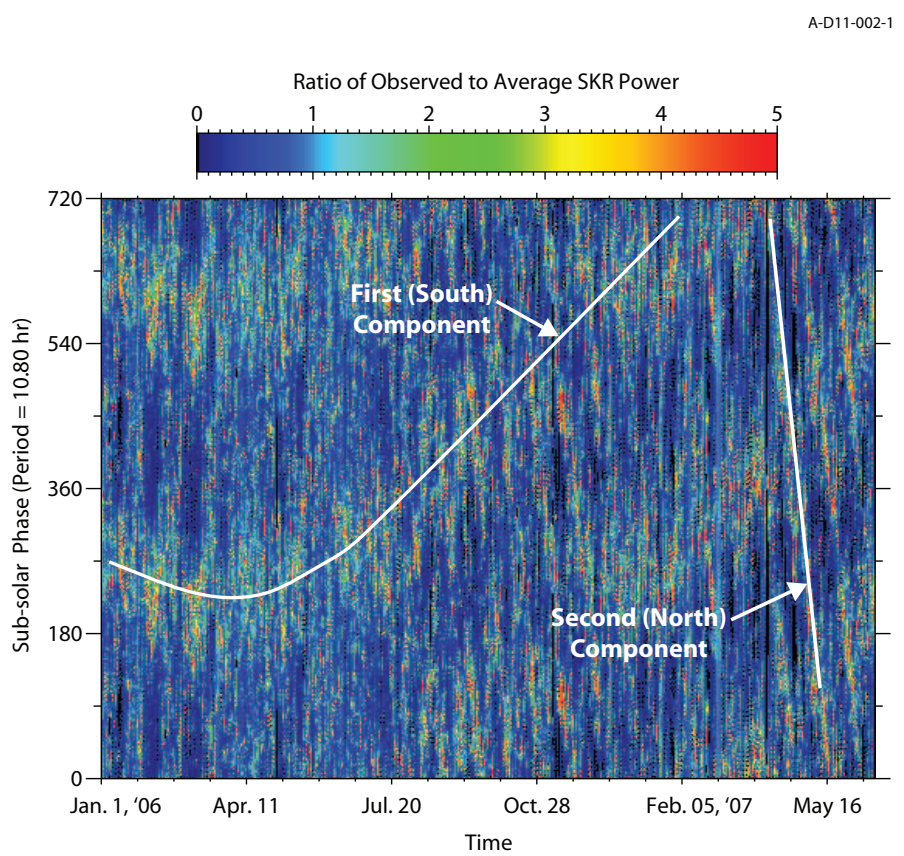

Figure 1: A plot of the SKR intensity as a function of subsolar longitude and time assuming a fixed period of 10.8 hours. The plot starts on 1 January 2006 and goes to 18 June 2007. Each rotation is plotted twice, so that the longitude varies from 0 to $720^{\circ}$. The "beat" patterns caused by the first (south) and second (north) components of the SKR are shown by the white lines. Illustration adapted from Kurth et al. [2008].

Although the two drift rates in Figure 1 are reasonably clear, sometimes it is difficult, or impossible, to clearly resolve the two components in such a plot. This is because the bandwidth involved is rather broad, wide enough to transmit both components of the modulation. The wide bandwidth introduces a considerable amount of background noise, which reduces the signal-to-noise ratio. What is needed is a narrowband filter that passes only the desired modulation component while suppressing the background noise to the maximum extent possible. The purpose of this paper is to describe a method of resolving the phase of the two SKR components by using a narrowband tracking filter that can separately track the modulation frequency of the two components. This technique is then used to define a new SKR based longitude system, one for the north that we call SLS4-N, and one for the south that we call SLS4-S. 


\section{SKR Modulation Spectrum}

Since the modulation rates of the two SKR components are separated by only about one percent, to resolve the two components it is necessary to have a spectrum analysis filter with a resolution, $\Delta \omega / \omega$, that is significantly less than one percent. Because of the constraint imposed on Fourier analysis by the uncertainty principle, $\Delta \omega \Delta t \sim 1$, the time resolution of the filter must necessarily be rather long, $\Delta t \sim 1 / \Delta \omega$. This means that the time window used in the spectrum analysis must be very long, at least several hundred times the modulation period, typically 100 days, or more. The exact choice of the filter bandwidth is necessarily a compromise. To improve the signal-to-noise ratio, and to give the best possible frequency resolution, the filter bandwidth should be as narrow as possible. On the other hand, if the bandwidth is too narrow, the rate of change of the modulation frequency, $\partial \omega / \partial \mathrm{t}$, can be so rapid that the frequency sweeps through the bandwidth of the filter in a time short compared to the response time of the filter. When this happens, as it sometimes does, then the signal-to-noise ratio is degraded. Since the variations in the modulation frequencies of the SKR are usually smooth and well defined, under these conditions a significant improvement in the signal-to-noise ratio can be achieved by using a filter that tracks the frequency variations (i.e., a tracking filter). In this section we describe a tracking filter technique that we have developed to improve the SKR modulation spectrum.

The first step in analyzing the SKR modulation is to integrate the electric field spectral density from the Cassini Radio and Plasma Wave Science (RPWS) instrument over a fixed frequency range to obtain a quantity that we call the intensity, in Watts $/ \mathrm{m}^{2}$. For a description of the RPWS see Gurnett et al. [2004]. Although the SKR spectrum sometimes extends from as low as $3 \mathrm{kHz}$ to as high as $1.2 \mathrm{MHz}$ [Kaiser et al., 1984], for the integration we have chosen to integrate from $80 \mathrm{kHz}$ to $500 \mathrm{kHz}$. The $80 \mathrm{kHz}$ low frequency limit was adopted to avoid contamination from narrowband radio emissions that sometimes occur below $80 \mathrm{kHz}$ [Ye et al., 2009], and the $500 \mathrm{kHz}$ upper frequency limit was adopted to avoid strong mode-dependent spacecraft generated interference lines that sometimes occur above this frequency. Two types of electric field measurements have been used. The first is from single $\mathrm{x}$-axis electric field measurements, and the second is from two- or three-axis electric field measurements. When the two- or three-axis measurements are being used the intensities are computed by integrating over the rightand left-hand circular polarization intensities after eliminating signals with a circular polarization degree, $\mathrm{v}$, less than 0.5 (i.e., $|\mathrm{v}|<0.5$ ). This later restriction is useful for eliminating spacecraft generated narrowband interference lines that tend to occur in some of the higher frequency channels, thereby increasing the signal-to-noise ratio. Generally, we found that if the SKR signal is strong the results obtained are not sensitive to the frequency limits on the integration, or the choice of antenna used. The one exception was near and shortly after the 11 August 2009 equinox when the northern SKR source was very weak. For this time period we used circular polarization measurements from the two- and three-axis measurements when processing signals from the second (north) component, since this gave a better signal-to-noise ratio. Otherwise, we use the $\mathrm{x}$-axis antenna measurements.

Since Cassini is in a highly eccentric orbit, periodic intensity variations occur that are 
caused by the $1 / R^{2}$ dependence on the radial distance from the planet. To minimize these orbit-induced periodicities, the measured intensities are normalized by dividing by a sliding average of the intensity averaged over one rotation of the planet, which was arbitrarily taken to be 10.79 hours. The next step in the processing is to perform the spectrum analysis and display the result in the form of a frequency-time spectrogram. To limit the spectrum analysis to a specific time window, $\Delta \mathrm{T}$, the normalized intensities are multiplied by a Hanning window function [Priestly, 1981] centered on a specific time, $\mathrm{T}_{n}$. The Hanning window function is a cosine function, see Figure 2, centered on $\mathrm{T}_{n}$ that goes to zero at the beginning and end of the window [Priestly, 1981]. The Hanning window function reduces the sideband response, and gives a filter response very close to an ideal

A-D 10-151-2

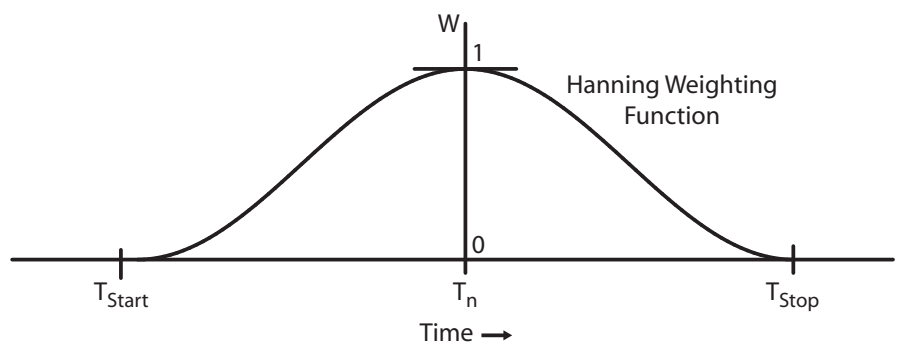

Figure 2: A plot of the Hanning weighting function used to restrict the spectrum processing to a specific time window, $\Delta T=T_{\text {Start }}-T_{\text {Stop }}$, centered on time $T_{n}$. The bandwidth of the resulting filter is approximately $\Delta \omega=1 /(2 \Delta T)$.

Gaussian filter. To a good approximation the effective bandwidth of the resulting filter is $\Delta \omega \sim 1 /(2 \Delta \mathrm{T})$, where $\Delta \mathrm{T}=\mathrm{T}_{\text {Start }}-\mathrm{T}_{\text {Stop }}$, is the duration of the Hanning window. We usually use a window duration of $\Delta \mathrm{T}=240$ days. This duration is a compromise between the desire to reduce the bandwidth (hence background noise), and the desire to track rapid frequency variations. To resolve zero-order temporal variations, the center of the Hanning window, $\mathrm{T}_{n}$, is advanced in 30 day steps, and an independent spectrum analysis is performed at each step. Since there are occasional gaps in the data a conventional Fourier analysis cannot be used. Instead the analysis is performed by sorting and averaging the SKR intensities into 1 degree sub-solar longitude bins for a series of assumed rotational modulation rates, $\omega$, where the longitude is given by $\lambda_{\text {Sun }}=\omega\left(\mathrm{t}-\mathrm{T}_{n}\right)$. For each $\omega$ the resulting averages, shown by red dots in Figure 3, are then fit to a cosine function of longitude, $A \cos \left[\left(2 \pi \lambda_{S u n} / 360^{\circ}\right)-\phi\right]$, shown by the blue line in Figure 3 . This fit gives the amplitude, A, and phase, $\phi$, of any modulation that might be present. The modulation power at time $\mathrm{T}_{n}$ and frequency $\omega$ is then taken to be proportional to the square of the peak-to-peak amplitude, $\mathrm{P}=(2 \mathrm{~A})^{2}$. To accurately determine the frequency of any peak that is present in the spectrum, the modulation power is then plotted as a function of the frequency, $\omega$, as in Figure 4, and the points around the peak are fit to a parabolic function in order to give a very accurate zero-order determination of the frequency of the peak, $\omega_{0}$. Using this procedure, the zero-order rotational modulation rates of the first (south) and second (north) components of the SKR can be determined and plotted as a 


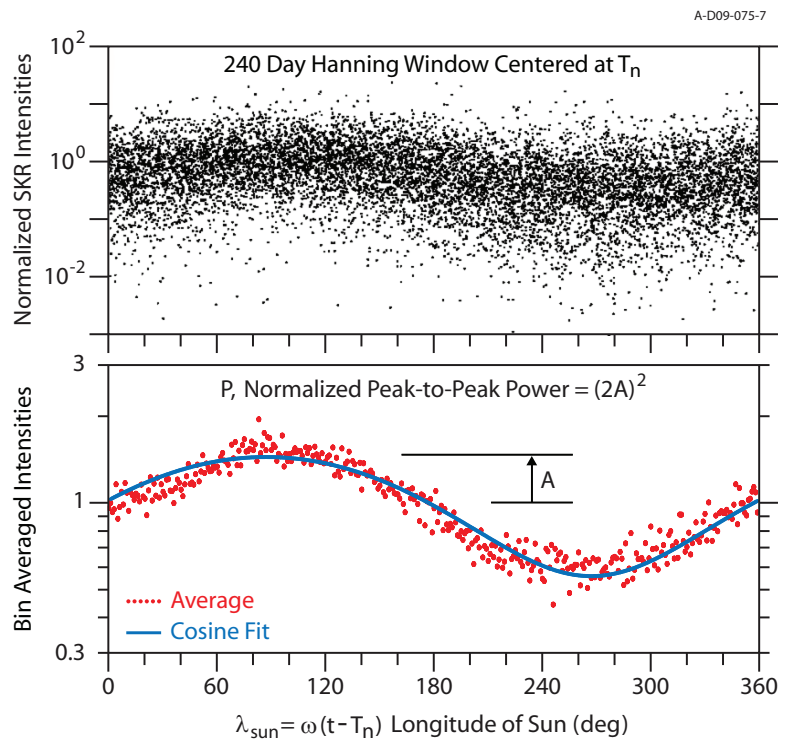

Figure 3: The top panel shows the normalized SKR intensities as a function of sub-solar longitude for an assumed rotational modulation rate, $\omega$. The bottom panel shows the average intensities in $1^{\circ}$ longitude bins (red) and the best fit cosine function (blue). The modulation power is taken to be the square of the peak-to-peak amplitude, $P=(2 \mathrm{~A})^{2}$.

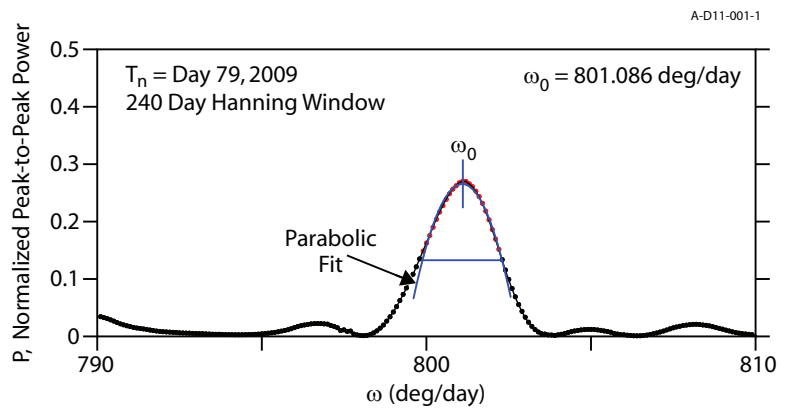

Figure 4: A plot of the modulation power, $P$, as a function of rotational modulation rate, $\omega$, for a spectrum around the first (south) component of the SKR centered on day 79, 2009. The parabolic fit procedure gives a very accurate determination of the peak, which in this case is at $\omega_{0}=801.086 \mathrm{deg} /$ day.

function of time, as shown in Figure 5.

From Figure 5 it can be seen that the rotational modulation rates of the two components remained relatively stable around $800 \pm 2$ and $816 \pm 2 \mathrm{deg} /$ day until late 2008, at 


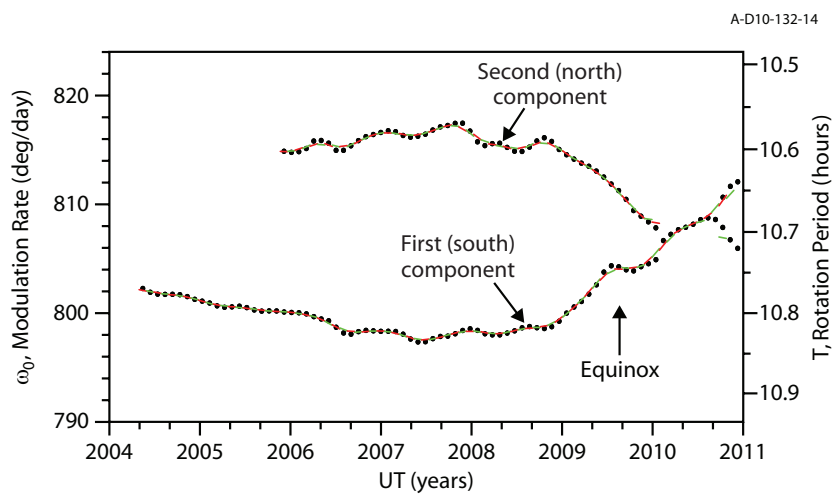

Figure 5: The best fit rotational modulation rates of the two SKR components plotted as a function of time in 30 day increments. The red and green lines give running five-point linear fits to the modulation rates. These lines give the zero-order slopes, $\left(\partial \omega_{0} / \partial t\right)$, used in the generation of the frequency-time spectrogram shown in Figure 6.

which point the two components began to rapidly converge, eventually crossing in lateFebruary/early-March 2010, shortly after the 11 August 2009 equinox [Gurnett et al., 2010]. In this region of rapid frequency change, $\partial \omega_{0} / \partial \mathrm{t} \sim 0.02 \mathrm{deg} / \mathrm{day}^{2}$, the signal no longer stays within the bandwidth of the spectrum analysis filter for the entire response time of the filter (sometimes as little as $30 \%$ ). This means that the signal-to-noise ratio is degraded because of the rapidly changing frequency. Since we know the relatively smoothly varying zero-order modulation frequencies of the north and south components, $\omega_{0}(\mathrm{~N})$ and $\omega_{0}(\mathrm{~S})$, this situation is ideally suited for using the tracking filter technique to improve the signal-to-noise ratio. The basic approach is to introduce a linear frequency correction that allows the signal to stay within the passband of the filter for the full duration of the Hanning window, thereby improving the signal-to-noise ratio. The first step in this process is to compute the rate of change of the zero-order frequencies, i.e., the slopes $\left(\partial \omega_{0} / \partial \mathrm{t}\right)$, at each time step in Figure 5 . This is done by computing the best fit straight line through five successive points centered on each time step. The resulting slopes are shown by the alternating red and green lines in Figure 5. It is easy to show that the longitude at each time step $\mathrm{T}_{n}$ is given to first-order by $\lambda_{\text {sun }}=\omega_{0}\left(\mathrm{t}-\mathrm{T}_{n}\right)$ $+(1 / 2)\left(\partial \omega_{0} / \partial \mathrm{t}\right)\left(\mathrm{t}-\mathrm{T}_{n}\right)^{2}$. Using this equation we then repeat the spectrum processing procedure described earlier using this new equation for the longitude. The $(1 / 2)\left(\partial \omega_{0} / \partial \mathrm{t}\right)(\mathrm{t}$ - $\left.\mathrm{T}_{n}\right)^{2}$ term introduces a frequency variation that to first-order tracks the zero-order frequency variations. Note that a separate spectrum analysis must be done for each of the two components since they have different slopes. The peak-to-peak modulation power as a function of time and frequency is then displayed in the form of a frequencytime spectrogram, as in Figure 6. In this plot the spectrums of the two components have been merged at a frequency intermediate between the two components. We have verified that this tracking filter approach gives a several dB improvement to the signalto-noise ratio in 2009 and early 2010 where the rotational modulation rates are changing rapidly. The signal-to-noise ratio improvement is especially important for the second 


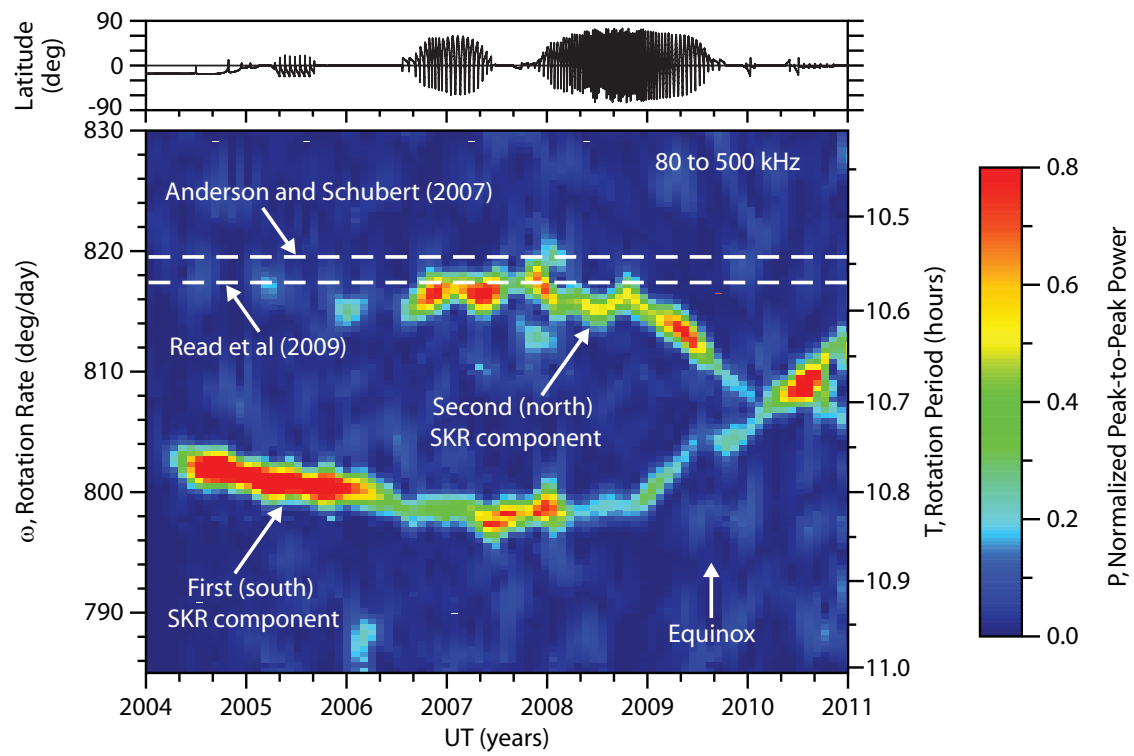

Figure 6: The top panel shows the latitude of Cassini, and the bottom panel shows a frequencytime spectrogram of the normalized peak-to-peak power of the SKR modulation as a function of Universal Time (UT) and the rotational modulation rate, $\omega$. The white dashed horizontal lines are the internal rotation rates of Saturn inferred by Anderson and Schubert [2007] and Read et al. [2009]. Note that the rotational modulation rate are all less than the inferred internal rotation rate which implies that the magnetospheric is slipping with respect to the rotation of Saturn's interior.

(north) component in late 2009 where the signal is very weak.

\section{Phase and Longitude Analysis}

Although the spectrum analysis procedure described above provides a high quality SKR modulation spectrum, it does not give the phase of the two components, which is necessary to define north and south longitude systems. In principle this phase information is contained in the cosine fitting procedure that is carried out every 30 days, as in Figure 3. However, this information is available only every 30 days, and we need to track the variations on time scales of minutes to hours. At this point there are several ways to proceed. The tracking technique that we have chosen is to integrate the zero-order frequencies (given in Figure 5) to give a zero-order estimate of the longitude for each of the two components, 


$$
\lambda_{0}=\int \omega_{0} d t+C,
$$

where a cubic spline fitting procedure [Forsythe et al., 1977] is used to interpolate the 30 day measurements to whatever time scale is needed. The constant $\mathrm{C}$ in equation (1) can be chosen only once. Two methods have been used to determine this constant. The first is adjust $\mathrm{C}$ so that the peak SKR intensity is at $\lambda=0^{\circ}$. The second is to add $100^{\circ}$ to this value in order to be consistent with the SLS2 and SLS3 longitude systems defined by Kurth et al. [2007, 2008], which has the peak SKR intensity at $\lambda=100^{\circ}$. Since the integration in equation (1) must be performed over time scales of years, errors are expected to accumulate in the integration process due to small errors in the zero-order frequencies. To correct for these accumulated errors the zero-order longitude is used in a cosine fitting procedure, comparable to that illustrated in Figure 3, in order to determine a phase correction, $\Delta \phi$, to the zero order longitude. The final phase corrected longitude is computed by subtracting the phase correction from the zero-order longitude.

$$
\lambda=\lambda_{0}-\Delta \phi
$$

To carry out the above procedure the first step is to perform the cubic spline fit to the zero order frequencies. The cubic spline fitting procedure involves simultaneously solving for a series of constant coefficients, $\omega_{0 n}, \mathrm{~A}_{n}, \mathrm{~B}_{n}$, and $\mathrm{C}_{n}$, in a cubic polynomial expansion

$$
\omega_{0}(t)=\omega_{0 n}+A_{n}\left(t-t_{n}\right)+B_{n}\left(t-t_{n}\right)^{2}+C_{n}\left(t-t_{n}\right)^{3}
$$

that matches the frequencies, $\omega_{0}(\mathrm{t})$, and slopes, $\partial \omega_{0} / \partial \mathrm{t}$, at each of the $\mathrm{n}$ points where the zero-order frequencies have been determined (i.e., from Figure 5). To perform the integration the above equation is integrated term by term to give a piecewise equation for the zero-order longitude

$$
\lambda_{0}(t)=\lambda_{n}+\omega_{0 n}\left(t-t_{n}\right)+A_{n}(1 / 2)\left(t-t_{n}\right)^{2}+B_{n}(1 / 3)\left(t-t_{n}\right)^{3}+C_{n}(1 / 4)\left(t-t_{n}\right)^{4}
$$

where the constants $\lambda_{n}$ must be chosen to give a smooth transition at each of the $\mathrm{n}$ points. The above equation is then used to fit the normalized intensities to $A \cos \left[\left(2 \pi \lambda_{0}(\mathrm{t}) / 360^{\circ}\right)\right.$ - $\phi$ ], as in Figure 7, to give the phase correction, $\Delta \phi$, to the zero-order longitude. Plots of the phase corrections as a function of time are shown in Figure 8 for the north and south components of the SKR. As can be seen, there are small but significant corrections to the zero-order longitudes caused by accumulated errors in the integration. These corrections are then subtracted from the zero-order longitudes using equation (2). The phase corrections tend to be largest for the second (northern) SKR component, apparently due to the more rapid and unpredictable fluctuations in this component, see Figure 6. The first (southern) component has a large, nearly $30^{\circ}$ negative correction in late 2009 . This correction appears to be related to the very weak modulation level and resulting poor signal-to-noise ratio present for this component in mid-2009, see Figure 6. 


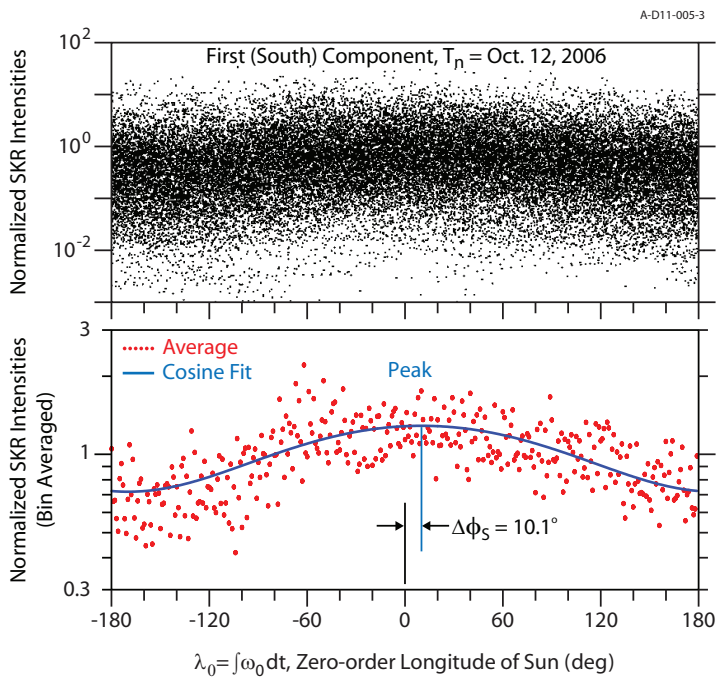

Figure 7: The top panel shows the SKR intensities as a function of the zero-order longitude, $\lambda_{0}=\int \omega_{0} d t$, and the bottom panel shows the resulting average intensities in $1^{\circ}$ longitude bins (red) and the best fit cosine function (blue). The phase difference $\Delta \phi$ is the correction to the zero-order longitude.

A-D10-149-4
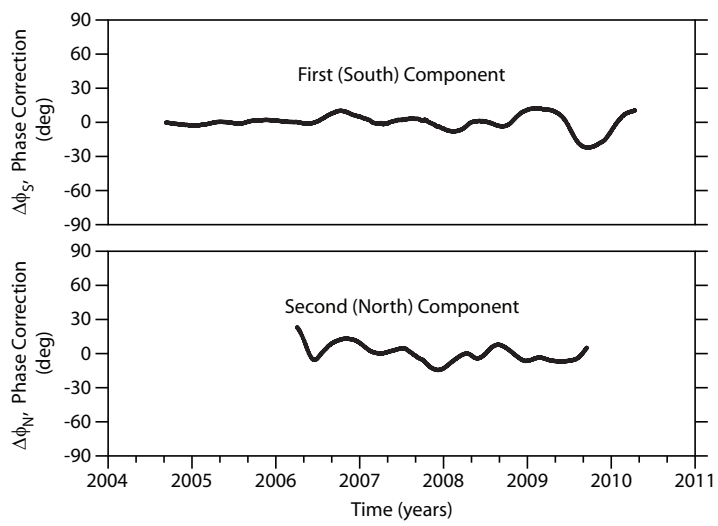

Figure 8: A plot of the phase correction, $\Delta \phi$, to the zero-order longitude, $\lambda_{0}=\int \omega_{0} d t$, as a function of time for the first (southern) component of the SKR in the top panel, and second (northern) components in the bottom panel. 


\section{The SLS4-N and SLS4-S Longitude Systems}

Using the tracking filter method described above we have proceeded to develop a webbased north and south SKR longitude system that can be accessed by anyone in the scientific community. As mentioned earlier, the integration constant, C, in equation (1) can be selected to place the peak SKR intensity at either $\lambda=0^{\circ}$ or $100^{\circ}$. To demonstrate how well this new system works we have constructed plots of the normalized SKR intensities as a function of the SLS4-N and SLS4-S longitudes and time, similar to Figure 1, with the peak at $100^{\circ}$ in order to be consistent with the earlier SLS2 and SLS3 longitude systems defined by Kurth et al. [2007, 2008] . These plots are shown in Figure 9. As can be seen the SLS4-N and SLS4-S longitude systems do a very good job of organizing the peak intensities along the white $100^{\circ}$ lines. The location of the peak intensities at $100^{\circ}$ can be demonstrated with even greater accuracy by processing the SKR intensities to give the longitude of the peak using the cosine fitting procedure illustrated in Figure 7 and displaying the results as a function of time. The results are shown in Figure 10 for three different Hanning window durations, 180, 240 and 300 days. As can be seen, the SLS4 longitude system maintains the peak longitude to within about \pm 3 to 5 degrees of $100^{\circ}$ for both the north and south components, independent of the filter parameters. Small phase oscillations can be seen with periods comparable to the response time of the filter, $\Delta \mathrm{t} \sim \Delta \mathrm{T} / 2$, i.e., 90,120 , and 150 days. These oscillations are believed to be due to excitation of the filter by fluctuations near the zero-order frequencies being tracked by the tracking filter. Possible causes of this "background noise" could be solar wind induced variations of the type discussed by Zarka et al. [2007], or some other yet unidentified variations in the Saturn system.

As a further test of the new longitude system we have made a plot of the difference between the SLS4-S longitude system and the SLS3 system defined by Kurth et al. [2008]. Note that the SLS3 system was based only on SKR from the first (south) source, so no comparison can be made with SLS4-N. The results are shown in Figure 11. As can be seen the two systems are in reasonably good agreement over the valid interval for the SLS3 system, beyond which large deviations occur, as would be expected. The deviations during the valid interval, sometimes as large as $\pm 45^{\circ}$, are almost certainly due to the fact that the polynomial expansion used by Kurth et al. [2008] cannot adequately follow some of the short-term variations that are present in the zero-order modulation.

\section{Accessing the SLS4 Longitude System}

The SLS4 longitude system can be accessed at the following web site:

http://www-pw.physics.uiowa.edu/sls4/. The longitude is in degrees using a west longitude system. Users should be prepared to specify (1) the time interval over which the longitudes are required, in either a year/month/day format (yyyy $/ \mathrm{mm} / \mathrm{dd}$ hh:mm:ss.sss) or in a year/day-of-year format (yyyy/ddd hh:mm:ss.sss); (2) the time resolution required, in seconds; (3) the longitude system desired, SLS4-North or SLS4-South; (4) the choice of subsolar longitude (Solar), or spacecraft longitude (spacecraft); and the choice of having the SKR peak intensity at either $0^{\circ}$ or $100^{\circ}$. 

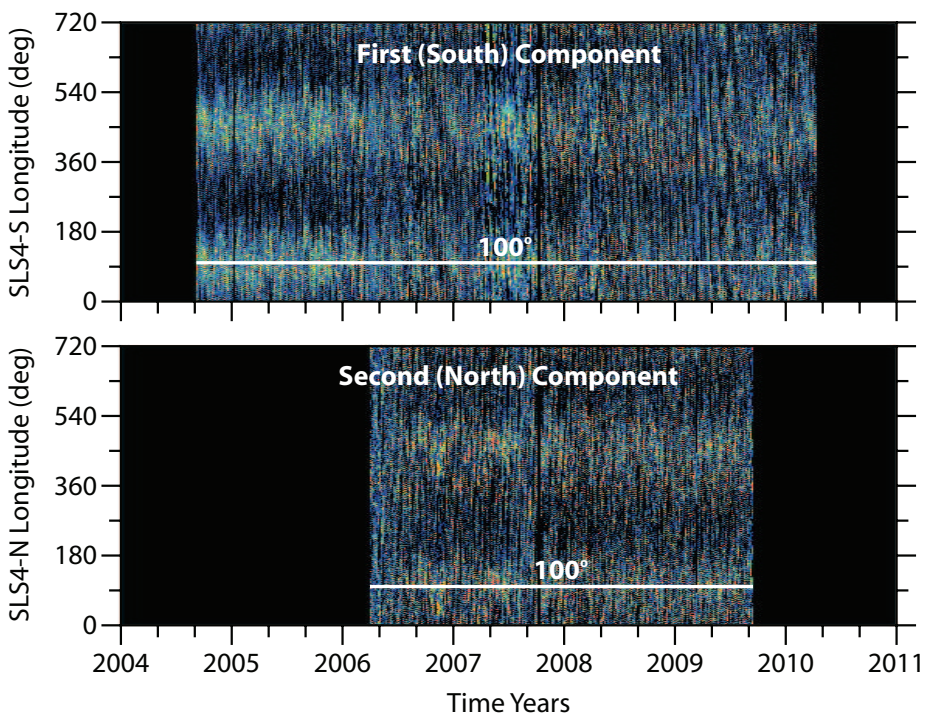

Figure 9: Plots comparable to Figure 1, showing the normalized SKR amplitude as a function of SLS4 longitude and time. The top panel is for the first (south) component, and the bottom panel is for the second (north) component. The horizontal white lines at $100^{\circ}$ longitude are the position of the peak SKR amplitude as defined for the SLS system by Seidelmann et al. [2002].
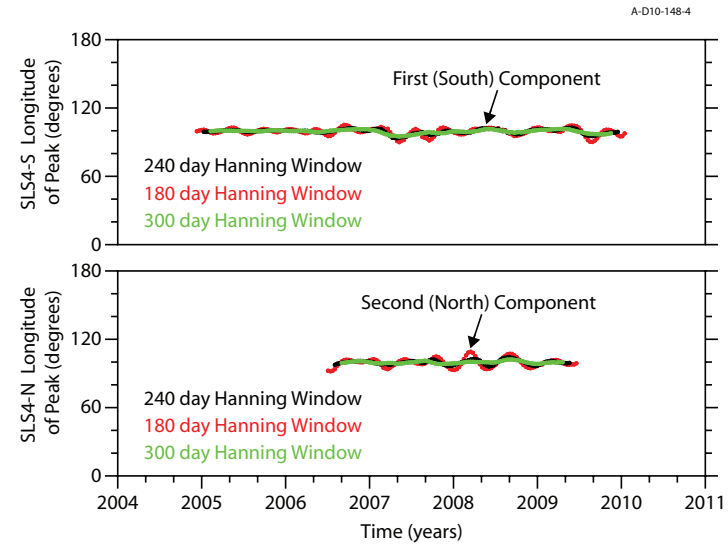

Figure 10: The top panel shows the SLS4 longitude of the peak SKR intensity for the first (south) component as a function of time, and the bottom panel shows a comparable plot for the second (north) component. The small oscillations are believed to be due to excitation of the tracking filter by "background noise" not associated with the zero-order modulation frequencies used in the tracking filter. 


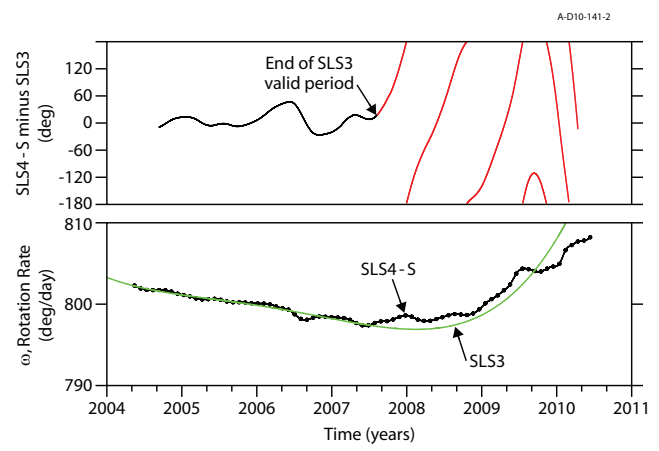

Figure 11: The top panel shows the difference between the SLS4-S longitude and the SLS3 longitude defined by Kurth et al. [2008]. The bottom panel shows a comparison of the rotational modulation rates for the two systems.

Acknowledgments. The research at the University of Iowa was supported by NASA through contract 1415150 with the Jet Propulsion Laboratory.

\section{References}

Anderson, J. D., and G. Schubert, Saturn's gravitational field, internal rotation, and interior, Science, 317, 1384-1387, 2007.

Davies, M. E., V. K. Abalakin, M. Bursa, J. H. Lieske, B. Morando, D. Morrison, P. K. Seidelmann, A. T. Sinclair, B. Yallop, and Y.S. Tjuflin, Report of the IAU/COSPAR working group on cartographic coordinates and rotational elements of the planets and satellites, Cel. Mech. Dyn. Astron., 63, 127-148, 1996.

Desch, M. D., and M. L. Kaiser, Voyager measurements of the rotation period of Saturn's magnetic field, Geophys. Res. Lett., 8, 253-256, 1981.

Forsythe, G.E., M. A. Malcolm, and C. B. Moler, Computer Methods for Mathematical Computations, Prentice-Hall, Englewood Cliffs, New Jersey, 70-79, 1977.

Galopeau, P., and A. Lecacheux, Variations of Saturn's radio rotation period measured at kilometric wavelengths, J. Geophys. Res., 105, A6, 13089-13102, 2000.

Gurnett, D. A., et al. (29 co-authors), The Cassini radio and plasma wave investigation, Space Sci. Rev., 114, 395-463, 2004.

Gurnett, D. A., et al. (26 co-authors), Radio and plasma wave observations at Saturn from Cassini's approach and first orbit, Science, 307, 1255-1259, 2005.

Gurnett, D. A., A. Lecacheux, W. S. Kurth, A. M. Persoon, J. B. Groene, L. Lamy, P. Zarka, and J. F. Carbary, Discovery of a north-south asymmetry in Saturn's radio rotation period, Geophys. Res. Lett., 36, L16102, doi:10.1029/2009GL039521, 2009. 
Gurnett, D. A., J. B. Groene, A.M. Persoon, J.D. Menietti, S.-Y. Ye, W.S. Kurth, R. J. MacDowall, and A. Lecacheux, The reversal of the north and south modulation rates of the north and south components of Saturn kilometric radiation near equinox, Geophys. Res. Lett., 37, L24101, doi;1029/2010GL045796, 2010.

Kaiser, M. L., M. D. Desch, J. W. Warwick, and J. B. Pearce, Voyager detection of nonthermal radio emission from Saturn, Science, 209, 1238-1240, 1980.

Kaiser, M. L., M. D. Desch, W.S. Kurth, A. Lecacheux, F. Genova, B. M. Pedersen, and D. R. Evans, Saturn as a radio source, in Saturn, edited by T. Gehrels, M. S. Matthews, 378-415, Univ. Arizona Press, Tucson, 1984.

Kurth, W. S., A. Lecacheux, T. F. Averkamp, J. B. Groene, and D. A. Gurnett, A Saturn longitude system based on a variable kilometric radiation period, Geophys. Res. Lett., 34, L02201, doi:10.1029/2066GL028337, 2007.

Kurth, W.S., T. F. Averkamp, D. A. Gurnett, J. B. Groene, and A. Lecacheux, An update to a Saturnian longitude system based on kilometric radio emissions, J. Geophys. Res., 113, A05222, doi:1029/2007JA012861, 2008.

Lecacheux, A., P. Galopeau, and M. Aubier, Re-visiting Saturnian kilometric radiation with Ulysses/URAP, in Planetary Radio Emissions IV, edited by H. O. Rucker, S. J. Bauer, and A. Lecacheux, 313-325, Austrian Academy of Science Press, Vienna, 1997.

Priestly, M. B., Spectral Analysis and Time Series, 443, Academic Press, London, 1981.

Read, P. L., T. E. Dowling, and G. Schubert, Saturn's rotation period from its atmospheric planetary-wave configuration, Nature, 460, 608-610, 2009.

Seidelmann, P. K., V.K. Abalakin, M. Bursa, M.E. Davies, C. de Bergh, J. H. Lieske, J. Oberst, J. L. Simon, E. M. Standish, P. Stoke, and P. C. Thomas, Report of the IAU/IAG working group on cartographic coordinates and rotational elements of the planets and satellites:2000, Cel. Mech. Dyn. Astron., 82. 83-110, 2002.

Ye, S.-Y., D. A. Gurnett, G. Fischer, B. Cecconi, J. D. Menietti, W. S. Kurth, Z. Wang, G. B. Hospodarsky, P. Zarka, and A. Lecacheux, Source locations of narrowband radio emissions detected at Saturn, J. Geophys. Res., 114, A06219, doi.1029/ 2008JA013855, 2009.

Zarka, P., L. Lamy, B. Cecconi, R. Prangé, and H. O. Rucker, Modulation of Saturn's radio clock by solar wind speed, Nature, 450, 265-267, 2007. 